Acta vet. scand. $1986,27,629-631$.

Brief Communication

\title{
CIRCUMSTANTIAL EVIDENCE OF AN EXTRACELLULAR (EPICELLULAR) LOCALIZATION OF DEVELOPMENTAL STAGES OF CRYPTOSPORIDIUM
}

Coccidian parasites of the genus Cryptosporidium have been reported in a variety of host species. Within the host, developmental stages of Cryptosporidium occur on the luminal surface of epithelial cells of the gastrointestinal and/or respiratory tract. The developing organisms are covered by an envelope of uniform thickness, consisting of 2 unit membranes and an intervening thin cytoplasmic layer. When observed in thin sections, the enveloped organisms appear to rest on a dense band (layer), on the surface of the host cells.

It is commonly held that both membranes of the envelope originate from the plasmalemma of the host cell microvilli. It has been suggested that the free zoite penetrates into the space between microvilli, inducing the adjacent microvilli to fuse with each other, thereby enclosing the parasite in a membrane-bounded vacuole (Brändler 1982, Marcial \& Madara 1986). Consequently, Cryptosporidium is regarded as an intracellular, but extracytoplasmic parasite.

Based on a detailed study of most of the published transmission electron micrographs (TEMs) of Cryptosporidium, I think the envelope is formed from the pellicle of the attaching zoite shortly after attachment to the host cell plasmalemma. Hence, Cryptosporidium is believed to be an extracellular (actually an epicellular) parasite.

The zoites of Cryptosporidium are limited by a pellicle consisting of 3 unit membranes (Brändler 1982, Marcial \& Madara 1986): an outer membrane or plasmalemma (M1), a middle membrane (M2), and an inner membrane (M3). M1 and M2 are separated by a thin cytoplasmic layer, whereas M2 and M3 are closely apposed and form the inner pellicular complex (Fig. 1, A). In low power TEMs the pellicle appears as 2 parallel dense lines, as the 2 membranes of the inner pellicular complex are visualized as a single dense line. The membranes of the envelope also appear as 2 parallel dense lines, and thus there should be 4 dense lines at the periphery of very early meronts, provided the zoites are enclosed by microvillar membranes. However, in a few published TEMs of presumably intracellular, early uninucleate meronts, the organisms have the appearance shown 


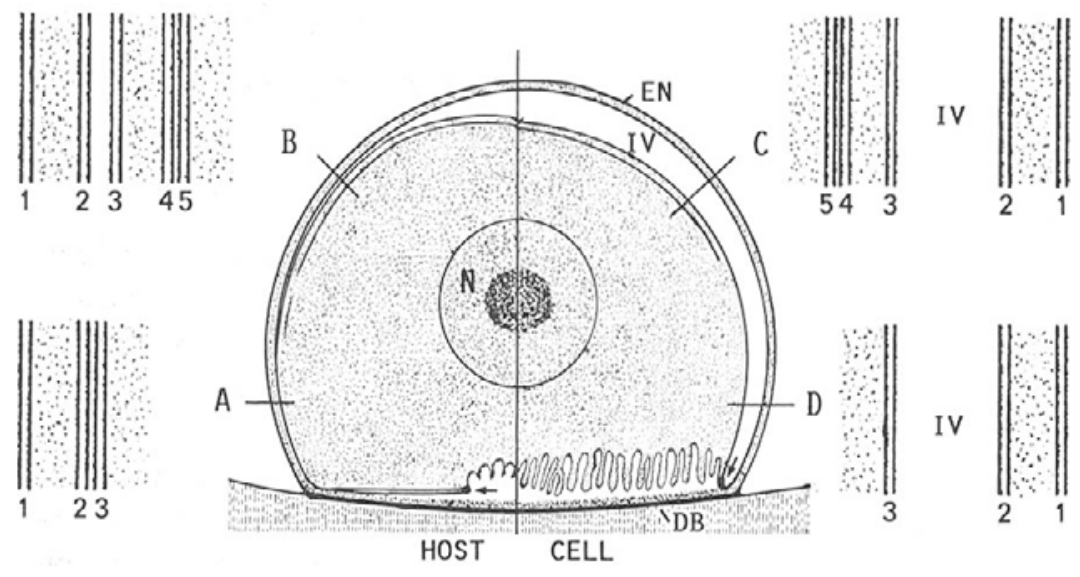

Figure 1. Schematic drawing of a section through a very early (left half), and a late (right half) uninucleate $(N)$ meront of Cryptosporidium, showing the proposed formation of the envelope (EN) from the pellicle of the attaching zoite. Sections A, B, C, and D show the relationship between the unit membranes (labelled $1-5$ ) of the pellicle/envelope in different stages of development. See text for details. IV $=$ intermembranal vacuole. $\mathrm{DB}=$ dense band. Arrows $=$ annular junction.

in the left half of Fig. 1. Thus, the developing organisms apparently are naked in some areas (2 dense lines), but covered by an envelope in other areas (4 dense lines). Yet, each of the 2 outer dense lines is continuous over the entire surface of the round bodies, irrespective of the number of dense lines, suggesting that the envelope arises from the pellicle. Moreover, the close similarity in thickness between the envelope and the pellicle of the zoites, irrespective of the stage of development or host species, strongly suggests that the envelope originates from the pellicle of the attaching zoite. Below, I will offer an explanation of how the envelope may be formed.

The zoite contacts the host cell membrane with its anterior end, and a circular zone of attachment arises, where the plasmalemma (M1) of the parasite becomes closely apposed to the plasmalemma of the host cell. Thus, the dense band seen in thin sections probably represents 2 closely apposed, or partially fused unit membranes, which form the parasite-host cell interface. Shortly after attachment, the closely apposed M2 and M3 of the original pellicle begin to separate ( 2 dense lines emerge from a single dense line, and 2 new and closely apposed membranes (M4 and M5) are formed interior to M3 of the original pellicle over most of the parasite's surface (Fig. 1, B). As the separation of M2 from M3 proceeds, narrow spaces arise between 
the 2 membranes focally. Eventually, M2 separates completely from M3, except around the base of the organism, and an intermembranal vacuole arises (Fig. 1, C). At the base of the organism, the 2 membranes join or remain together, and thus form an annular junction (Fig. 1, arrows).

The annular junction is located quite near the center of the attachment area of the very early uninucleate meront, suggesting it might be a relic of the anterior polar ring of the attaching zoite, the structure where M2 and M3 of the inner pellicular complex of the zoite terminate. If this is so, it will explain the absence of $\mathrm{M} 2$ in the central portion of the attachment area, where no membrane covers the thin cytoplasmic layer above the dense band. However, this implies that the highly folded plasmalemma at the base of the developing organism does not originate from M3, even though the folded membrane seems to be continuous with M3 at the annular junction. The 2 newly formed membranes, M4 and M5, subsequently disappear, except in the apical portion of the meront (Fig. 1, C and D).

Thus, according to the present theory, M1 and M2 of the original pellicle become the outer and inner membrane of the envelope, respectively, whereas M3 of the original pellicle becomes the plasmalemma of the developing meront/gamont, except perhaps at its base. In addition, a new inner pellicular complex (M4 and M5) is formed interior to M3. Consequently, Cryptosporidium is thought to be an extracellular (epicellular) parasite which develops within itself, the pellicle-derived envelope being an integral part of the organism.

\section{Bjørn Gjerde}

The Division of Parasitology, Department of Internal Medicine I, Norwegian College of Veterinary Medicine, Norway.

\section{REFERENCES}

Brändler, $U$ : Licht- und elektronenmikroskopische Untersuchungen der Entwicklung von Cryptosporidium sp. im Darm experimentell infizierter Mäuse. (Light and electron microscopic studies on the development of Cryptosporidium sp. in the intestine of experimentally infected mice). Vet. med. diss., München 1982, $55 \mathrm{pp}$.

Marcial, M. A. \& J. L. Madara: Cryptosporidium: Cellular localization, structural analysis of absorptive cell-parasite membrane-membrane interactions in guinea pigs, and suggestion of protozoan transport by M cells. Gastroenterology $1986,90,583-594$.

(Received December 18, 1986).

Reprints may be requested from: B. Gjerde, the Norwegian College of Veterinary Medicine, P. O. Box 8146 Dep., N-0033 Oslo 1, Norway. 